\title{
The Effects of Radiation Feedback on Early Fragmentation and Stellar Multiplicity
}

\author{
Stella S. R. Offner ${ }^{1}$ \\ ${ }^{1}$ Harvard-Smithsonian Center for Astrophysics, 60 Garden St, Cambridge MA 02138, USA \\ email: soffner@cfa.harvard.edu
}

\begin{abstract}
Forming stars emit a significant amount of radiation into their natal environment. While the importance of radiation feedback from high-mass stars is widely accepted, radiation has generally been ignored in simulations of low-mass star formation. I use ORION, an adaptive mesh refinement (AMR) three-dimensional gravito-radiation-hydrodynamics code, to model lowmass star formation in a turbulent molecular cloud. I demonstrate that including radiation feedback has a profound effect on fragmentation and protostellar multiplicity. Although heating is mainly confined within the core envelope, it is sufficient to suppress disk fragmentation that would otherwise result in low-mass companions or brown dwarfs. As a consequence, turbulent fragmentation, not disk fragmentation, is likely the origin of low-mass binaries.
\end{abstract}

Keywords. stars:formation, binaries, hydrodynamics, turbulence, radiative transfer

\section{Introduction}

The origin of stellar multiplicity remains an unsolved problem in star formation. The dense conditions in molecular cloud cores and dim luminosities of young protostars make estimations of the initial multiplicity distribution challenging (Duchêne et al. 2007). However, the present-day multiplicity can be observed among field stars, where the likelihood of companions is strongly correlated with the primary stellar mass. Nearly all $\mathrm{O}$ and $\mathrm{B}$ stars are found in binaries or multiple systems, while only $\sim 20 \%$ of M stars have companions (Lada 2006). Successful simulations and theories of star formation must be able to predict the multiplicity fraction and explain why it depends so strongly on stellar mass.

A number of mechanisms have been proposed of which two appear to have the most potential for producing the observed number of multiple systems (see Tohline 2002 for a review). First, gravitational instability within a protostellar accretion disk may produce companions within a few 100 AU (Adams et al. 1989; Bonnell et al. 1994). Repeated fragmentation over the disk lifetime may generate numerous companions. Second, perturbations within a turbulent core may seed additional fragmentation on scales of $\sim$ 0.001-0.1 pc (Fisher 2004; Goodwin et al. 2004). This must occur within the first $\sim 0.5$ Myr of collapse, when the core still contains at least $0.1 \mathrm{M}_{\odot}$.

In this paper, we discuss the effect of radiation feedback on early fragmentation and stellar multiplicity using 3D adaptive mesh refinement (AMR) simulations of turbulent molecular clouds. We compare the cases with and without radiation from forming stars. We describe the simulations in $\S 2$, present results in $\S 3$, and conclude in $\S 4$.

\section{Simulations}

The ORION code solves the equations of compressible gas dynamics, Poisson equation, and radiation energy equation in the flux-limited diffusion approximation (Krumholz 
et al. 2007):

$$
\begin{aligned}
\frac{\partial \rho}{\partial t}+\nabla \cdot(\rho \mathbf{v}) & =0 \\
\frac{\partial(\rho \mathbf{v})}{\partial t}+\nabla \cdot(\rho \mathbf{v v}) & =-\nabla P-\rho \nabla \phi, \\
\frac{\partial(\rho e)}{\partial t}+\nabla \cdot[(\rho e+P) \mathbf{v}] & =\rho \mathbf{v} \nabla \phi-\kappa_{R} \rho(4 \pi B-c E), \\
\frac{\partial E}{\partial t}-\nabla \cdot\left(\frac{c \lambda}{\kappa_{\mathrm{R}} \rho} \nabla E\right) & =\kappa_{\mathrm{P}} \rho(4 \pi B-c E)+\sum_{n} L_{n} \delta\left(\mathbf{x}-\mathbf{x}_{n}\right), \\
\nabla^{2} \phi & =4 \pi G\left[\rho+\sum_{n} m_{n} \delta\left(\mathbf{x}-\mathbf{x}_{n}\right)\right],
\end{aligned}
$$

where $\rho, P, \mathbf{v}$ and $e$ are the fluid density, pressure, velocity, and specific kinetic plus internal energy of the gas, $\phi$ is the gravitational potential, $m_{n}, \mathbf{x}_{n}$, and $L_{n}$ are the mass, position, and luminosity of the $\mathrm{n}^{\text {th }}$ star, $E$ is the radiation energy density, and $\kappa_{\mathrm{R}}$ and $\kappa_{\mathrm{P}}$ are the Rosseland and Planck dust opacities. For comparison, a second calculation closes the equations with a barotropic equation of state (EOS) in lieu of equation 2.4:

$$
P=\rho c_{\mathrm{s}}^{2}+\left(\frac{\rho}{\rho_{\mathrm{c}}}\right)^{\gamma} \rho_{\mathrm{c}} c_{\mathrm{s}}^{2},
$$

where $c_{\mathrm{s}}=\left(k_{\mathrm{B}} T / \mu\right)^{1 / 2}$ is the sound speed, $\gamma=5 / 3$, the average molecular weight $\mu=2.33 m_{\mathrm{H}}$, and the critical density, $\rho_{\mathrm{c}}=2 \times 10^{-13} \mathrm{~g} \mathrm{~cm}^{-3}$.

The calculations insert Lagrangian sink particles in regions exceeding the Jeans density on the maximum AMR level (Krumholz et al. 2004). In the simulation with radiation, the particles are endowed with a sub-grid model based upon McKee \& Tan (2003) that includes the accretion energy, Kelvin-Helmholtz contraction, and nuclear burning (Offner et al. 2009).

The calculations have a Mach number of $\mathcal{M}_{3 \mathrm{D}}=6.6$, domain size $L=0.65 \mathrm{pc}$, and mass $M=185 M_{\odot}$, which correspond to an approximately virialized cloud. We adopt periodic boundary conditions for the gas and Marshak boundary conditions for the radiation field, which allows the cloud to cool. We use a $256^{3}$ base grid with 4 levels of grid refinement, where $\Delta x_{4}=32$ AU. High-resolution convergence tests are discussed in detail in Offner et al. (2009). We drive the boxes for three crossing times using random velocity perturbations with wavenumbers $1 \leqslant k \leqslant 2$ after which self-gravity is turned on. The initial gas temperature is $10 \mathrm{~K}$. Since the gas cools efficiently during the driving phase, the radiation calculation remains nearly isothermal.

\section{Results}

\subsection{Fragmentation}

At the end of a freefall time, the two calculations have very different temperature distributions. Temperatures in the EOS calculation do not exceed $15 \mathrm{~K}$, while temperatures in the radiation calculation reach $\sim 100 \mathrm{~K}$. In the latter, because the heated cores are turbulent, a range of densities is heated to various temperatures such that gas temperature is not a single valued function of gas density. The heating also varies with the number of stars and their instantaneous accretion rate, such that any EOS fit to a mean temperature-density curve would be a generally poor approximation over the course of the simulation.

As a consequence of the feedback, the calculation with radiation has a fundamentally different mass distribution (Figure 1A). The protostellar heating is sufficient to raise the temperature of the gas significantly within a few hundred AU and suppress fragmentation 

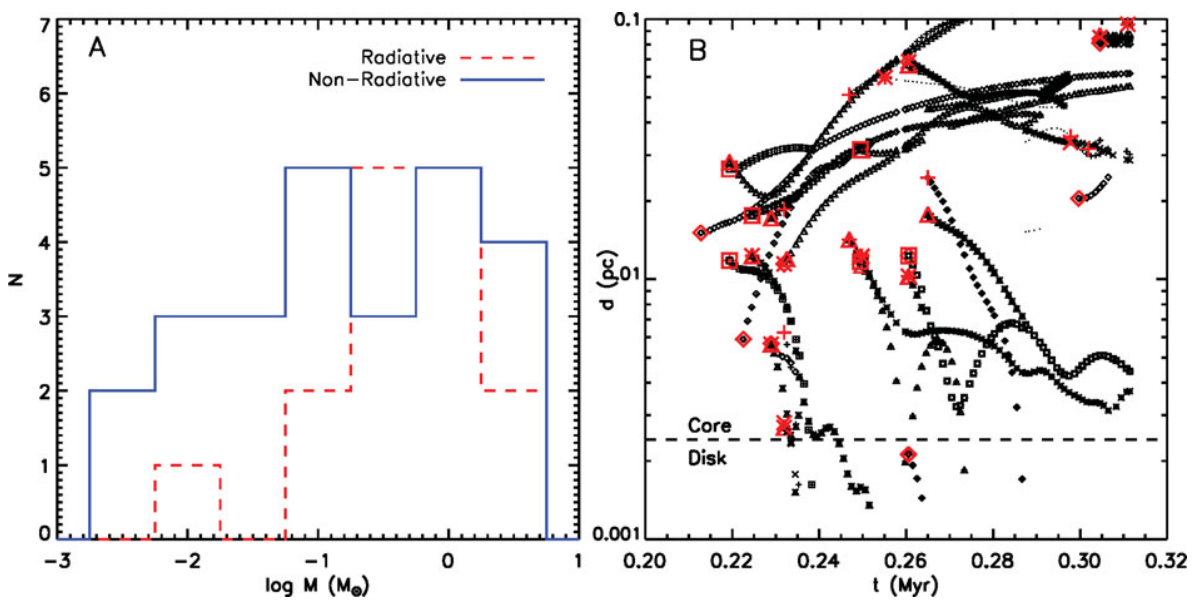

Figure 1. Left: Distribution of star masses produced from simulations with (dashed) and without (solid) radiative feedback. Right: Pair separation as a function of time in $1 \mathrm{kyr}$ bins for all particle pairs from Offner et al. (2010). The dashed line at $500 \mathrm{AU}$ indicates a rough boundary between the disk and core scales. The large majority of pairs have separations $>0.1 \mathrm{pc}$ and are not shown. The large (red) symbols indicate the first time bin.

in accretion disks that would otherwise be unstable. As a result, the radiative calculation has far fewer brown dwarfs and, despite small number statistics, its mass distribution more closely resembles the stellar initial mass function. Bate (2009) has previously demonstrated similar results using smoothed-particle hydrodynamics simulations.

Although the heating is efficient at small scales, it is limited to the parent core and does not inhibit fragmentation of other cores or even wide fragmentation within the same core. Figure 1B shows the separations of all star pairs as a function of time in the calculation with radiation feedback. The plot is restricted to separations of $0.1 \mathrm{pc}$ or less to highlight stars forming within the same core that may comprise a binary. The plot shows that fragmentation on scales of $\sim 2000$ AU is not suppressed by heating. The one instance of fragmentation with $d<500 \mathrm{AU}$ is actually filament rather than disk fragmentation. This suggests that turbulent core fragmentation remains a viable mechanism for binary formation of low-mass stars, while disk fragmentation is much less likely.

\subsection{Disk Analysis}

Although the disks in the simulations are not well resolved, it is still possible to use their mean properties to draw robust conclusions about the protostellar multiplicity. Kratter et al. (2010) define a two-dimensional parameter space for characterizing accretion and disk stability:

$$
\xi=\frac{\dot{M}_{\mathrm{in}} G}{c_{\mathrm{s}, \mathrm{d}}^{3}}, \quad \Gamma=\frac{\dot{M}_{\mathrm{in}}}{M_{* \mathrm{~d}} \Omega_{\mathrm{k}, \text { in }}}=\frac{\dot{M}_{\mathrm{in}}\langle j\rangle_{\mathrm{in}}^{3}}{G^{2} M_{* \mathrm{~d}}^{3}},
$$

The thermal parameter, $\xi$, compares the core sound speed to the disk sound speed, $c_{\mathrm{s}, \mathrm{d}}$, where $\dot{M}_{\text {in }}$ is the infall mass accretion rate. A collapsing isothermal sphere has $\xi \simeq 1$ (Shu 1977). For $\xi>1$, a disk will be unable to efficiently process accreting material and will eventually fragment. The rotational parameter, $\Gamma$, compares the disk orbital time to the gas infall time, where $M_{* \mathrm{~d}}$ is the total mass in the star-disk system, $\Omega_{\mathrm{k} \text {,in }}$ is the Keplerian angular velocity at the circularization radius of the infall, and $\langle j\rangle_{\text {in }}$ is the specific angular momentum. For large $\Gamma(\Gamma \sim 0.1)$ the disk mass changes quickly over an orbital time.

In Figure 2 we estimate these parameters for the disks in each calculation. Our analysis confirms that disks in the radiative calculation are stable, where the binaries to the left 


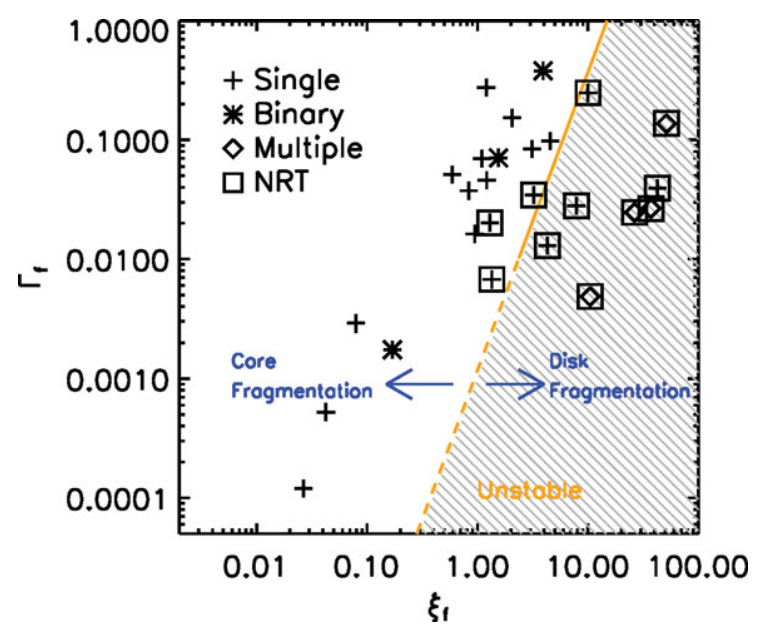

Figure 2. The values of $\Gamma$ and $\xi$ at 1 freefall time for the protostellar disks in each simulation, where the Non-Radiative Transfer (NRT) cases are denoted by boxes. The diagonal line indicates the boundary between stable and unstable disks found by Kratter et al. (2010).

of the line are in fact products of turbulent rather than disk fragmentation. Without radiation feedback large unstable disks yield high multiplicity systems, which fall to the right of the line as expected. Although some single systems exist within the unstable regime these tend to have low $\mathrm{Q}$ values and often have previously fragmented.

\section{Conclusions}

Radiation feedback from low-mass stars is important in shaping the stellar mass distribution. Heating works to stabilize protostellar disks and suppress fragmentation that would otherwise over-produce brown dwarfs. However, fragmentation of the parent core may still occur on thousand AU scales, suggesting that turbulent core fragmentation, not disk fragmentation, is the most likely origin of low-mass binaries.

\section{References}

Adams, F. C., Ruden, S. P., \& Shu, F. H. 1989, ApJ, 347, 959

Bate, M. R. 2009, MNRAS, 392, 1363

Bonnell, I. A. \& Bate, M. R. 1994, MNRAS, 269, L45

Duchêne, G., Delgado-Donate, E., Haisch, Jr., K. E., Loinard, L., \& Rodríguez, L. F. 2007, Protostars and Planets V, 379

Fisher, R. T. 2004, ApJ, 600, 769

Goodwin, S. P., Whitworth, A. P., \& Ward-Thompson, D. 2004, A\& A, 414, 633

Kratter, K. M., Matzner, C. D., Krumholz, M. R., \& Klein, R. I. 2010, ApJ, 708, 1585

Krumholz, M. R., McKee, C. F., \& Klein, R. I. 2004, ApJ, 611, 399

Krumholz, M. R., Klein, R. I., McKee, C. F., \& Bolstad, J. 2007, ApJ, 667, 626

Lada, C. J. 2006, ApJL, 640, L63

McKee, C. F., \& Tan, J. C. 2003, ApJ, 585, 850

Offner, S. S. R., Klein, R. I., McKee, C. F., \& Krumholz, M. R. 2009, ApJ, 703, 131

Offner, S. S. R., Kratter, K. M., Matzner, C. D., Krumholz, M. R., \& Klein, R. I. 2010, in prep.

Shu, F. H. 1977, ApJ, 214, 488

Tohline, J. E. 2002, ARAA, 40, 349 